This is the peer reviewed version of the following article: Gholizadeh Leila et al. 2010, 'Theoretical considerations in reducing risk for cardiovascular disease: implications for nursing practice', Blackwell Publishing Ltd, vol. 19, no. 15, pp. 2137-2145. which has been published in final form at http://dx.doi.org/10.1111/j.1365-2702.2009.03189.x This article may be used for non-commercial purposes in accordance With Wiley Terms and Conditions for self-archiving' 
TITLE

\title{
THEORITICAL CONSIDERATIONS IN REDUCING RISK FOR CARDIOVASCULAR DISEASE: IMPLICATIONS FOR NURSING PRACTICE
}

Descriptive title of the paper: Perception of risk of CVD

\author{
Leila Gholizadeh MSc, RN \\ PhD Candidate \\ School of Nursing \\ University of Western Sydney \\ Sydney, Australia \\ Tabriz University of Medical sciences
Patricia M. Davidson PhD, RN
Professor
Cardiovascular and Chronic Care
Curtin University of Technology
University of Western Sydney
Australia \\ Yenna Salamonson PhD, RN \\ Senior Lecturer \\ School of Nursing \\ University of Western Sydney \\ Sydney, Australia

\section{Linda Worrall-Carter PhD, RN} \\ Professor \& Director \\ St Vincent's/ACU National Centre for Nurse Research \\ School of Nursing \& Midwifery (Victoria) \\ Faculty of Health Sciences \\ Australian Catholic University \\ Melbourne, Australia
}

Corresponding Author:

Yenna Salamonson PhD, RN

Senior Lecturer

School of Nursing, College of Health \& Science

University of Western Sydney

Campbelltown Campus, Building 7

Locked Bag 1797

Penrith South. DC 1797

New South Wales, Australia

Phone: +6124620 3322

Fax: $\quad+61246203161$

Email:y.salamonson@uws.edu.au

The number of words: 3476 


\section{ABSTRACT}

INTRODUCTION: Understanding how to maximize cardiovascular health is a focus of clinicians, researchers, and policy makers.

AIMS AND OBJECTIVES: This article presents a theoretical perspective of the perception of risk as a key component of behaviour change models and discusses this perspective in relation to cardiovascular disease risk reducing behaviours within a socio-cultural model.

METHODS: An integrative review method was used to appraise evidence on the perception of risk of developing cardiovascular disease. On the basis of the findings, a conceptual model was developed to inform future intervention studies.

DISCUSSION: Findings demonstrated that many people consistently underestimate their personal risk of developing cardiovascular disease. Studies examining cardiovascular disease and health behaviours report that there is limited concordance between actual and perceived risk in people with cardiovascular disease. This mismatch between actual and perceived risk likely impedes the adoption of risk reducing behaviours.

CONCLUSION: There is a critical need to improve an accurate perception of personal cardiovascular disease risk considering not only individual but social factors. 


\section{INTRODUCTION}

Cardiovascular disease (CVD) refers to the class of diseases that involves the heart or blood vessels(Cecil et al., 2000) and is a cause of disease burden in both developed and developing countries.(Reddy et al., 1998) The major manifestations of CVD are coronary heart disease (CHD), stroke, and peripheral arterial disease, which share common risk factors and often develop together.(Cecil et al., 2000) Yet, more than half of the deaths and disability from CVD can be prevented through public health initiatives and the actions of individuals to reduce modifiable CVD risk factors, such as high blood pressure, high blood cholesterol, and smoking.(Davidson et al., 2004) Although the modifiable risk factors for CVD are generally well identified and consistent among most populations throughout the world,(Rosengren et al., 2004) risk factor modification, which primarily involves behaviour change, is a complex and multifaceted phenomenon. Pivotal to modifying risk reducing behaviours is the accurate perception of risk.(Janz, 1998) Risk perception is subjective judgments and evaluations of individuals of potential hazards.(Slovic et al., 2004) Through influencing individuals' perceptions and beliefs about their CVD risk, health care professionals can increase the chance of people adopting healthier lifestyles.(Garrity et al., 1985) This also includes not only considering individual factors but also the socio-cultural factors impacting on risk perception and behaviours.(Leventhal et al., 1992) The perception of susceptibility to risk influences individual's reactions and behaviours.(Davison et al., 1991;

Zabsonre et al., 2002) Understanding factors that impact upon the individual's capacity to accurately perceive their risk and balance the negative and positive aspects of changing their behaviours can assist health professionals in their clinical practice and inform intervention studies.

\section{AIMS AND OBJECTIVES}

This article aimed to present theoretical underpinnings of risk perception as a critical component in health behaviour models and discuss how these could impact on the individual's capacity to engage 
in affirmative CVD risk reducing behaviours. In particular this review has attempted to explore models that may have greater relevance to cultures where there is a greater emphasis on collective values, attitudes and beliefs in to societies where there is an emphasis on individualism.(Towle et al., 2003) The questions driving this integrative review related to investigating the: (1) theoretical underpinnings of risk perception; (2) factors impacting upon the perception of the risk of CVD; and (3) the interventions most likely to improve the accuracy of risk perception and effect CVD behaviour change.

\section{METHODS}

An integrative review method was used to present the state of perception of risk of CVD in the literature and derive a more comprehensive understanding of the concept. An integrative review is a type of literature review that summarizes both past experimental and non-experimental literature to obtain a more comprehensive understanding of a phenomenon under study.(Sparbel et al., 2000; Whittemore, 2005) A literature search was conducted using Medline, CINAHL, and EMBASE databases. The World Wide Web was also searched using the Google Search Engine. The key terms used in this search were 'cardiovascular disease', 'perception of risk', and 'behaviour change models'. We did not limit our search to specific time periods. Articles were chosen for this review if they addressed the theoretical underpinnings of risk perception in general and/or investigated risk perception specifically in relation to CVD as well as interventions to promote accurate perception of CVD risk. Publications in languages other than English were excluded form the review.

\section{RESULTS}

In spite of the importance of addressing behaviour change in influencing health outcomes, health and risk behaviours are not well understood and there is evidence of the poor congruence between knowledge and consequential behaviours.(Weinstein, 1999; Wilcox et al., 1999) Adverse 
consequences of unhealthy lifestyles are manifested in conditions such as cardiovascular disease, diabetes, and cancer, thus many health researchers are becoming interested in adopting and applying behaviour change models to better understand those factors associated with maximizing healthy behaviours. Perception of risk has been a key element of many theoretical perspectives and models, notably, the Health Belief Model (HBM), the Leventhal's Self-Regulatory Model of Health and Behaviours, and the Extended Parallel Process Model. These models will be discussed in further details below.

\section{The Health Belief Model}

The Health Belief Model (See Figure 1) was originally developed in the early 1950's as a method to explain and predict individuals’ health behaviours, and has been subsequently revised to include illness role behaviours.(Becker et al., 1975) The fundamental components of the HBM are the individual's perceived susceptibility to and the severity of a given disease, their perception of the barriers and benefits to reduce the risk, and recently added components including cues to action and self-efficacy.(Janz, 1998) The HBM states that the perception of risk is influenced by at least three factors:

- General health values;

- Beliefs about vulnerability to a particular health threat;

- Beliefs about the consequences of the given health problem.(Becker et al., 1975)

The Health Belief Model has been successfully applied and tested in a number of studies aimed at understanding an individuals' willingness to adopt health behaviours as well as actual behaviours change. A comprehensive review of these studies concluded perceived risk as an important predictor of most preventive health behaviours.(Janz et al., 1984) Yet, predictability of perception of risk in taking risk reducing behaviours after developing a disease such as a cardiovascular event 
has not been as powerful as preventive health behaviours. For example, screening for hypertension or attending a cardiovascular secondary prevention program.(Janz, 1998) Although the HBM focuses on risk perception as a key element in behaviour change, perception of risk and factors influencing a person's perceived susceptibility to a threat are not transparently defined in this model.

\section{Leventhal's Self-Regulatory Model of Health and Illness}

The Self-Regulatory Model of Health and Illness, developed by Howard Leventhal(Leventhal et al., 1999) in 1970, considers that two parallel and interactive processes including cognition and emotion are active when confronting with a health threat. (See Figure 2)These two parallel process influence the representation of the threat which include causal attributions, labelling the symptoms, the perception of temporality and curability, and the ability to control events and the consequences of a particular condition.(Leventhal et al., 1992) Internal and external factors, such as demographic attributes, health history, personality, culture, and social environment also influence an individual's illness representation. These factors individually and collectively contribute to an individual's capacity to cope with a health threat and take action to reduce the risk.(Cherrington et al., 2004; French et al., 2002; Leventhal et al., 1992) Perceived control of illness and symptoms largely determines the individual's appraisal changes. ${ }^{17}$

Leventhal's Self-Regulatory Model has been tested in a number of studies aiming to link between peoples' perception of risk and actions taken to control stress and reduce the risk, such as attendance at CR in patients after a cardiovascular event.(Cherrington et al., 2004; French et al., 2002) When facing a health threat such as myocardial infarction, a person forms a representation of a heart attack which guides their identification and utilisation of available services, such as seeking treatment in order to control the consequences.(Dracup et al., 1995) Nevertheless, studies have often examined the predictive role of only one or two components of the Leventhal's SelfRegulatory Model, such as causal attributions and have not tested the model comprehensively.(Byrnea et al., 2005; Dracup et al., 1995; MacInnes, 2006) An important aspect of 
this model is considering the emotional response as well as cognitive recognition in risk representation and also considering factors beyond the individual, such as social and environment elements which impact upon both cognitive and emotional processes. The emotional processes initiate emotion related coping strategies to control stress, which are influenced by socio-cultural factors, such as belief in fatalism, while cognitive processes initiate cognitive coping strategies, which help effective decision making and problem solving to manage the risk such as engaging in risk reducing behaviours.(Leventhal et al., 1992) Jayne et al(Jayne et al., 2001) applied Leventhal’s Self-Regulation Model to Chinese immigrants with type 2 diabetes and found that emotional process related to socio-cultural values about food and eating appeared to be more significant than cognitive processes. Davison et al(Davison et al., 1991) argued that certainly socio-cultural factors impact upon the individual's representation of CVD risk, causal attributions, and their strategies to control the risk.(Davison et al., 1991; Hodoglugil et al., 2000; Murray et al., 2000) To date, the predominance of either the cognitive or emotional processes of the Leventhal's model in culturally and linguistically diverse groups have been poorly studied.

\section{The Extended Parallel Process Model}

The Extended Parallel Process Model (See Figure 3) developed by Witte(Witte, 1992) in 1992 provides a valuable conceptual foundation for describing the predictive role of perception of risk in behaviour change. It suggests that self-efficacy and response efficacy should be assessed in parallel with risk perception. According to this model, a high perception of risk along with low self-efficacy and/or low response efficacy may result in maladaptive responses.(Gordon, 2002) Self efficacy refers to self-effectiveness and response efficacy refers to response effectiveness.(Witte et al., 2001) For example, a person who perceives a higher risk of developing heart disease, but believes heart disease has solely a heredity basis and is not related to modifiable risk factors will likely perceive a low response efficacy from the modifiable risk factors and as a consequence may persist with these behaviours. According to this model, the social environment also influences an individual's health 
behaviour decisions. The Extended Parallel Process Model further considers perceived costs and benefits as the other factors affecting the likelihood of adopting health behaviours. From an individual's point of view, if the costs outweigh the benefits, pre-existing behaviours are more likely to persist.(Gordon, 2002) This dialectical process undertaken by the individual is important in engaging in positive health-seeking behaviours. This model may have particular applicability to cross cultural populations, where extrinsic factors often play a greater role in risk perception. For example, in Australian Aborigines, long-standing social disadvantage may fuel a lower self-efficacy and potential to change behaviour, as this may be perceived as futile.(Farley et al., 2003)

\section{Perception of risk in risk reducing behaviours: A socio-cultural model}

Presented in Figure 4 is a conceptual model drawn from the literature review illustrating the role of perception of risk in risk reducing behaviours. This conceptual model is a hybrid approach, incorporating elements of the Health Belief Model (HBM), Leventhal's Self-Regulatory Model of Health and Behaviours, and the Extended Parallel Process Model. This conceptual model also provides factors influencing an individual's perception of risk, both objective and experiential. These factors has drawn from the literature investigating the perception of risk of cardiovascular disease and therefore provides an excellent platform to assist health professionals to improve accuracy of risk perception with regard to CHD which also facilitates risk reducing behaviours.

According to the literature, some factors impacting on perception of risk of CHD include age,(Meischke et al., 2000) gender,(Moran et al., 1989) knowledge,(Kaplan et al., 1996; Wilcox et al., 1999) cultural beliefs and values,(Bottorff et al., 2004; Davison et al., 1991) psychological status,(Cameron et al., 2005) general health status,(Green et al., 2002) pre-existing heart disease,(Fiandt et al., 1999; Green et al., 2002) family history of heart disease,(Erblich et al., 2003) the visibility of risk factors,(Zabsonre et al., 2002) and the number of risk factors.(Silagy et al., 1993) Perception of risk(Davison et al., 1991; Glanz et al., 2002; Zabsonre et al., 2002) along with 
self efficacy and response efficacy(Bandura, 1977; Gordon, 2002; Janz et al., 1984) have been considered as internal resources and important determinants of health-related behaviour change. High level of perception of risk with low level of self efficacy and response efficacy have been associated with rejection of a recommended health behaviour.(Witte, 1992) Health messages; therefore, not only should stimulate an appropriate level of risk perception but also consider the individual's perceptions of their ability to take a recommended behaviour (self efficacy) as well as the efficacy of the recommend behaviour (response efficacy). The extent to which people are engaged in risk reducing behaviours is also influenced by external resources, both socio-cultural and instrumental, such as support that one receives from spouse and significant others and availability of appropriate health services.(Chyun et al., 2003; Palacios et al., 2005; Toobert et al., 2002) A final factor affecting the likelihood of adopting healthful behaviours is weighing the perceived barriers and benefits. Behaviour change is more likely to occur when the perceived benefits outweigh the perceived costs.(Becker et al., 1975)

The conceptual model proposes that an accurate perception of susceptibility to CHD risk, belief in effectiveness of CHD risk reducing behaviours, and perceived higher self efficacy in relation to risk factor modification as well as a supportive socio-environment increase the likelihood of adopting a risk reducing behaviour. Bandura's (Bandura, 1977) Social Cognitive Theory explains that there is a continual reciprocal interaction between the individual, environment, and their behaviours. As discussed earlier, self-efficacy refers to the individual's ability to perform a behaviour or set of behaviours to achieve a desired outcome.(Bandura, 1977) A range of factors impact on developing self-efficacy including performance accomplishment, vicarious experience, verbal persuasion and physiological feedback.(Bandura, 1977) Further, a range of views of social support have been put forward. Social support can be emotional and/or instrumental.(Wilson et al., 1999) This means individuals modify their behaviour to fit the socio-cultural values, emphasising the importance of considering these factors in interaction. 
The status of perceived personal risk of developing CVD in the literature is addressed which set the context to discuss about the applicability of the conceptual model to improve cardiovascular health.

\section{Perception of personal CVD risk}

It is plausible that if people perceive their risk of a given disease accurately they will make changes in their risk taking behaviours.(Newman, 2004; Weinstein, 1999) Yet, misperception of one's personal CHD risk has consistently been reported.(Cooper et al., 2000; Fiandt et al., 1999; Legato et al., 1997; Mosca et al., 2004a) people, particularly women often tend to underestimate their heart risk.(Fiandt et al., 1999; Legato et al., 1997) In one study, 73\% of the respondents estimated their risk of developing heart disease by age 70 years to be less than $1 \%$, and surprisingly twice as many women reported being worried about breast cancer than the risk of heart disease.(Pilote et al., 1995) Further, an international study which included 5 European countries(Erhardt et al., 2002) reported that only $45 \%$ of the public correctly identified heart disease as the leading cause of death in their country. In Australia, as few as $10 \%$ of women believed that CVD was the primary killer of women over 45 years.(Davidson et al., 2004) Those who underestimate their CVD risk tend to have lower incomes, education, and anxiety levels.(Moran et al., 1989) Further, using the Framingham risk score, Christian and colleagues(Christian et al., 2005) compared ethnically diverse women's perceived risk with their estimated risk of developing CVD. This study demonstrated that the estimated risk of developing CVD over 10 years was low in 59\% of the participants. When compared with participants' perceived risk, only 52\% correctly perceived their risk of CVD as being low. The study concluded that most women with a lower risk overestimated their chances, but young women who were at moderate or high risk often underestimated their risk of developing CVD. This incongruence has been also reported between actual and perceived underlying risk factors of CVD. For example, Murphy and colleagues(Byrnea et al., 2005) found a mismatch between Australian women's identified CVD risk factors and their perceptions of the underlying 
causes of their condition. The highest discrepancy between actual and perceived CVD risk factors was in hypertension, with as few as $5 \%$ of women with a positive history of high blood pressure acknowledging hypertension as a possible cause of their heart problems. This lack of congruence between actual and perceived risk is important and should be taken into account when developing effective CVD prevention and management strategies.

\section{Improving the accuracy of cardiovascular risk perception}

Studies have reported the amenability of risk perceptions and suggested that both primary and secondary prevention programmes should be designed to stimulate appropriate levels of concerns while also considering the receivers' beliefs about themselves and the recommended behaviour.(Johnston et al., 1992; Pimm et al., 1994) These interventions have improved screening for breast cancer and vaccination.(Klein et al., 2007) However, relatively few studies have examined the success of interventions in changing perceptions regarding CHD risk. While some interventions have failed to improve risk perception and health outcomes,(Cooper et al., 2006) others successfully led to show any effect on improving risk perception leading to improving health outcomes such as attendance at CR.(Petrie et al., 2002)

A single educational session designed to improve attendance at CR through correcting misperceptions and improving illness perception of patients after MI failed to show any difference in attendance at CR between intervention and control group.(Cooper et al., 2006) Further, a brief educational intervention was successful in changing perception of personal risk of developing heart disease in ethnically diverse women without heart disease (125 women) who were chosen from those who underwent mammography screening.(Christian et al., 2005) Immediately after the educational intervention there was a significant increase in the proportion of women who correctly categorised their risk as low (67\% in the intervention group vs. 52\% in the control group). However, after one month, only 52\% of participants in intervention group correctly perceived their risk as low. 
Further, among moderate and high risk women, with absolute risk equal and greater than 10\%, there was a steady decline in the number of women who accurately perceived their risk $(71 \%$, $68 \%$, and 64\% respectively).(Christian et al., 2005) Outcome measurement of this study was simply recalling the information and the question of whether interventions at this level could lead to behaviour change was not answered by the above study.

The results of these studies show that simple education is not adequate to improve risk perception for a long term and suggest that interventions to modify perceived risk should account for many other factors such as one's health beliefs and values. Petrie et al.(Petrie et al., 2002) applied a randomised controlled trail to examine an in-hospital intervention to change cardiac patients' perceptions regarding their disease. The intervention group received the standard care plus three brief visits by a psychologist. The first visit focused on discussion of the causes of the myocardial infarction, the second visit focused on what the patient could do to minimise future risk, and the third visit aimed to develop an action plan. At discharge, there was a significant difference between the intervention and control group in their beliefs about the consequences of their myocardial infarction, controllability of the disease, and whether they could control the future course of their disease. The simple intervention also influenced return to work in that patients with strong feelings that cardiac disease did not have serious consequences and believed that their illness would not last too long were more likely to return to work.

\section{DISCUSSION}

Trends in public awareness and knowledge of cardiovascular disease are increasing in developed countries which mean that more people know heart disease is a serious health threat. According to a recent report from the American Heart Association, 57\% of women surveyed in 2006 were aware of heart disease as their leading cause of death compared to 46\% reported in 2003.(American Heart Association, 2006; Mosca et al., 2004b; Mosca et al., 2000) Without doubt, national awareness 
campaigns have had an important role in improving awareness of heart in the USA, such as 'go red for women'.(American Heart Association, 2007) Although the improvement in public awareness of heart disease as a major health threat is a great achievement, concerns remain as people fail to use this information to formulate a judgment of their personal vulnerability to CHD. The percentage of women who perceived heart disease as their major health threat has increased steadily, with $7 \%$ in 1997 and $8 \%$ in $2000 \%$ to $13 \%$ in 2004.(Mosca et al., 2004b) These data suggests that changing perceptions, particularly when it comes to one's personal risk is far more difficult than changing an individual's knowledge and as discussed earlier risk perception is a subjective, complex and multifaceted phenomenon.(Weinstein, 1999) Interventions should focus on convincing people that they are at greater personal risk than they think, and should take a proactive role to protect their cardiovascular health.(Giorgianni, 2003) an effective and individualised risk communication which account for factors influencing a person's perceptions of vulnerability to a risk such as discussing about one's risk factors are more likely to improve risk perceptions and narrow the gap between actual and perceived personal risk. Some of CVD risk assessment tools such as the Framingham Risk Score could provide a more realistic picture of a person's absolute and relative risks and improve accuracy of perceived personal risk.(Bussoletti, 2003) Also, more recently the Heart Age Patient Tool(Goldman et al., 2006) has been developed and evaluated as potentially capable of assisting people to develop a more realistic risk perception and motivating health-related risk reducing behaviours.

Interventions to improve risk perceptions should also include discussions about invisible risk factors such as high blood cholesterol level, as people tend to underestimate the significance of risk factors that are not visible.(Zabsonre et al., 2002) Further, disputably, the receptivity and the capacity to accept one's personal risk and change behaviours are influenced by an individual's beliefs and culture. For instance, in some cultures a strong belief in fatalism influences attitudes and practices toward prevention measures and treatment.(Manson, 1988) 


\section{Limitations}

Limitations of this review study include: first, the first author of the study selected and reviewed the literature alone. Second, the search was limited to literature published only in English language. Third, the literature on factors influencing perception of risk included distinctively publications that examined risk perception in relation to cardiovascular disease.

\section{FUTURE DIRECTIONS}

The existing studies on perception of risk and the contributing factors have yielded inconsistent and sometimes ambiguous results.(Meischke et al., 2000; Missik, 1999; Moran et al., 1989; Wilcox et al., 1999) This is partially because of the inherent difficulties experienced in measuring subjective concepts.(Weinstein, 1999) Using well designed studies with larger sample sizes may better describe factors associated with CVD risk perceptions. Furthermore, previous studies have either applied quantitative or qualitative approaches to explain the concept of perception of risk. Given the complexity of the concept of perception of risk,(Weinstein, 1999) using a mixed method approach may yield a better explanation of risk perception and the contributing factors, likely leading to developing models and theories that specifically focus on risk perception.

\section{RELEVANCE TO CLINICAL PRACTICE}

As discussed above, the accurate perception of risk is complex and extends far beyond purely providing information. Understanding the factors impacting on the accurate perception of CVD risk has significant implications for the implementation of effective nursing interventions. Targeting groups at higher risk for inaccurate risk perception, such as those from culturally and linguistically diverse groups, may improve primary, secondary and tertiary CVD strategies. Nurses are in an excellent position to assess the accuracy of risk perception and should consider this factor when providing education and counseling strategies. 


\section{CONCLUSION}

Findings of this review study underscore a need to improve an accurate perception of personal cardiovascular disease risk among people, considering not only individual but social factors. Health professionals including nurses are in a unique position to assess the accuracy of risk perception when providing education and counseling strategies. 


\section{References}

American Heart Association. (2006). Awareness of cardiovascular disease increasing among women. Available at: www.americanheart.org/presenter.jhtml?identifier=3037182. (accessed January 14, 2007).

American Heart Association. (2007). Go red for women. Available at: http://www.goredforwomen.org/. (accessed October 12, 2007).

Bandura, A. (1977). Self-efficacy: Toward a unifying theory of behavioral change. Psychological Review, 84(2), 191-215.

Becker, M. H., \& Maiman, L. A. (1975). Sociobehavioral determinants of compliance with health and medical care recommendations. Medical care, 13, 10-24.

Bottorff, J. L., Richardson, C., Balneaves, L. G., McCullum, M., Buxton, J. A., Ratner, P. A., et al. (2004). Unrevealing women's perceptions of risk for breast cancer Health Education Research, 19(4), 469-475.

Bussoletti, A. (2003). Framingham point score: A useful tool in assessing CHD risk. Journal of the American Academy of Nurse Practitioners, 7(9), 17-20.

Byrnea, M., Walsha, J., \& Murphy, A. W. (2005). Secondary prevention of coronary heart disease: Patient beliefs and health-related behaviour. Journal of Psychosomatic Research, 58, 403- 415.

Cameron, L. D., Petrie, K. J., Ellis, C., Buick, D., \& Weinman, J. A. (2005). Symptom experiences, symptom attributions, and causal attributions in patients following firsttime myocardial infarction. International Journal of Behavioral Medicine, 12(1), 3038.

Cecil, R. L., Claude Bennett, J., \& Goldman, L. (Eds.). (2000). Cecil textbook of medicine (21st ed.). Philadelphia: W.B. Saunders Company.

Cherrington, C. C., Moser, D. K., Lennie, T. A., \& Kennedy, C. W. (2004). Illness representation after acute myocardial infarction: Impact on in-hospital recovery. American Journal of Critical Care, 13(2), 136-145.

Christian, A. H., Mochari, H. Y., \& Mosca, L. J. (2005). Coronary heart disease in ethnically diverse women: Risk perception and communication. Mayo Clinic Proceedings, 80(12), 1593-1599.

Chyun, D., Amend, A., Newlin, K., Lagerman, S., \& Melkus, G. (2003). Coronary heart disease prevention and lifestyle interventions: Cultural influences. The Journal of cardiovascular nursing, 18(4), 302-318.

Cooper, A., Weinman, J., Chalder, T., Jackson, G., \& Horne, R. (2006). An illness perceptions intervention to address cardiac misconceptions and increase attendance at cardiac rehabilitation post myocardial infarction: Results of a pilot randomised controlled trial. Circulation. 2006;114:II_466-II_467.), 114, 466-467.

Cooper, R., Cutler, J., Desvigne-Nickens, P., Fortmann, S. P., Friedman, L., Havlik, R., et al. (2000). Trends and disparities in coronary heart disease, stroke, and other cardiovascular diseases in the United States: Findings of the national conference on cardiovascular disease prevention. Circulation, 102, 3137-3147.

Davidson, P., Daly, J., Hancock, K., \& Jackson, D. (2004). Australian women and heart disease: Trends, epidemiological perspectives and the need for a culturally competent research agenda. Contemporary Nurse, 16, 62-73.

Davison, C., Smith, G., D., \& Frankel, S. (1991). Lay epidemiology and the prevention paradox: The implications of coronary candidacy for health education. Sociology of health \& illness, 13(1), 329-340. 
Dracup, K., Moser, D. K., Eisenberg, M., Meischke, H., Alonzo, A. A., \& Braslow, A. (1995). Causes of delay in seeking treatment for heart attack symptoms. Social science \& medicine, 40(3), 379-392.

Erblich, J., Bovbjerg, D. H., Norman, C., Valdimarsdottir, H. B., \& Montgomery, G. H. (2003). It won't happen to me: Lower perception of heart disease risk among women with family histories of breast cancer. Preventive Medicine, 31(6), 714-721.

Erhardt, L., \& Hobbs, F. D. (2002). Public perceptions of cardiovascular risk in five European countries: The react survey. International Journal of Clinical Practice, 56(9), 638644.

Farley, R. L., Wade, T. D., \& Birchmore, L. (2003). Factors influencing attendance at cardiac rehabilitation among coronary heart disease patients. European Journal of Cardiovascular Nursing, 2(3), 205-212.

Fiandt, K., Pullen, C. H., \& Walker, S. N. (1999). Actual and perceived risk for chronic illness in rural older women. Clinical Excellence for Nurse Practitioners, 3(2), 105115.

French, D. P., Marteau, T. M., Senior, V., \& Weinman, J. (2002). The structure of beliefs about the causes of heart attacks: A network analysis. British Journal of Health Psychology, 7, 463-479.

Garrity, T. F., \& Garrity, A. B. (1985). The nature and efficacy of intervention studies in the national high blood pressure education research program. Journal of Hypertension, 3, S91-S95.

Giorgianni, S. J. (2003). Through the doors of perception and reality about cardiovascular disease. The Pfizer Journal, VII(4), 1.

Glanz, K., Lewis, F. M., \& Rimer, B. K. (2002). Health behavior and health education: Theory, research, and practice (3rd ed.). San Francisco: Jossey- Bass.

Goldman, R. E., Parker, D. R., Eaton, C. B., Borkan, J. M., Gramling, R., Cover, R. T., et al. (2006). Patients' perceptions of cholesterol, cardiovascular disease risk, and risk communication strategies. Annals of Family Medicine, 4(4), 371-371.

Gordon, J. C. (2002). Beyond knowledge: Guidelines for effective health promotion messages. Journal of Extension, 40(6).

Green, J. S., Hill, K. L., \& Grant, M. (2002). Physical activity and heart disease risk perception in young college women. Research Quarterly for Exercise and Sport, 73(1), Supplment: A-24.

Hodoglugil, N. N. S., Sommerfeld, J., \& Gursoy, A. (2000). Understanding the risk perception in cardiovascular health and disease.Unpublished manuscript, Ankara.

Janz, N. K. (1998). The health belief model in understanding cardiovascular risk factor reduction behaviors. Cardio-vascular nursing, 24(6), 39-41.

Janz, N. K., \& Becker, M. H. (1984). The health belief model: A decade later. Health Education Quarterly, 11(1), 1-47.

Jayne, R. L., \& Rankin, S. H. (2001). Application of Leventhal's Self-Regulation Model to Chinese immigrants with type 2 diabetes. Journal of nursing scholarship, 33(1), 5359.

Johnston, M., Gilbert, P., Partridge, C., \& Collins, J. (1992). Changing perceived control in patients with physical disabilities: An intervention study with patients receiving rehabilitation. The British journal of clinical psychology, 31(Pt 1), 89-94.

Kaplan, G. A., Goldberg, D. E., Everson, S. A., Cohen, R. D., Salonen, R., Tuomilehto, J., et al. (1996). Perceived health status and morbidity and mortality: Evidence from the Kuopio ischaemic heart disease risk factor study. International Journal of Epidemiology, 25(2), 259-265. 
Klein, W. M., \& Stefanek, M. E. (2007). Cancer risk elicitation and communication: Lessons from the psychology of risk perception. CA: A Cancer Journal for Clinicians, 57(3), 147-167.

Legato, M. J., Padus, E., \& Slaughter, E. (1997). Women's perceptions of their general health, with special reference of their risk of coronary heart disease: Results of national telephone survey. Journal of women's health, 6, 189-198.

Leventhal, H., Diefenbach, M., \& Leventhal, E. A. (1992). Illness cognition: Using common sense to understand treatment adherence and affect cognition interactions. Cognitive therapy and research, 16, 143-163.

Leventhal, H., Kelly, K., \& Leventhal, E. A. (1999). Population risk, actual risk, perceived risk, and cancer control. Journal of the National Cancer Institute, 91(18 ), 81.

MacInnes, J. D. (2006). The illness perception of women following symptoms of acute myocardial infarction: A self regulatory approach. European Journal of Cardiovascular Nursing, 5, 280-288.

Manson, A. (1988). Language concordance as a determinant of patient compliance and emergency room use in patients with asthma. Medical care, 26, 1119-1128.

Meischke, H., Sellers, D. E., Robbins, M. L., Goff, D. C., Daya, M. R., Meshack, A., et al. (2000). Factors that Influence personal perceptions of the risk of an acute myocardial infarction. Behavioral Medicine, 26(1), 4-13.

Missik, E. (1999). Personal perceptions and women's participation in cardiac rehabilitation. Rehabilitation Nursing, 24, 158 -165.

Moran, M. T., Mazzocco, V. E., Fiscus, W. G., \& Koza, E. P. (1989). Coronary heart disease risk assessment. American Journal of Preventive Medicine, 5(6), 330-336.

Mosca, L., Appel, L. G., Benjamin, E., J., Berra, K., Chandra-Strobos, N., Fabunmi, R. P., et al. (2004a). Evidence-based guidelines for cardiovascular disease prevention in women. Circulation, 109, 672- 693.

Mosca, L., Ferris, A., Fabunmi, R., \& Robertson, R. M. (2004b). Tracking women's awareness of heart disease: An American heart association national study. Circulation, 109(5), 573- 579.

Mosca, L., Jones, W. K., King, K. B., Ouyang, P., Redberg, R. F., \& Hill, M. N. (2000). Awareness, perception, and knowledge of heart disease risk and prevention among women in the United States. Archives of Family Medicine, 9, 506-515.

Murray, S. A., Manktelow, K., \& Clifford, C. (2000). The interplay between social and cultural context and perceptions of cardiovascular disease. Journal of Advanced Nursing, 32(5), 1224-1233.

Newman, S. (2004). Engaging patients in managing their cardiovascular health. Heart, 90, iv9.

Palacios, J., Butterfly, R., \& Strickland, C. J. (2005). American Indians. In J. G. Lipson \& S. L. Dibble (Eds.), Culture \& Clinical Care (pp. 28). San Francisco: UCSF Nursing Press.

Petrie, K. J., Cameron, L. D., Ellis, C. J., Buick, D., \& Weinman, J. (2002). Changing illness perceptions after myocardial infarction: An early intervention randomized controlled trial. Psychosomatic Medicine, 64, 580-586.

Pilote, L., \& Hlatky, M. (1995). Attitudes of women toward hormone therapy and prevention of heart disease. American Heart Journal, 129, 1237-1238.

Pimm, T. J., Byron, M. A., \& Curson, D., et al. . (1994). Personal illness models and the selfmanagement of arthritis Arthritis and Rheumatism, 37(suppl), 9.

Reddy, K. S., \& Yusuf, S. (1998). Emerging epidemic of cardiovascular disease in developing countries. Circulation, 97, 596-601. 
Rosengren, A., Hawken, S., Ôunpuu, S., Sliwa, P., Zubaid, M., Almahmeed, W. A., et al. (2004). Association of psychosocial risk factors with risk of acute myocardial infarction in 11119 cases and 13648 controls from 52 countries (the INTERHEART study): Case-control study Lancet, 364(9438), 953-962

Silagy, C., Muir, J., Coulter, A., Thorogood, M., \& Roe, L. (1993). Cardiovascular risk and attitudes to lifestyle: What do patients think? British Medical Journal, 7(307), 380381.

Slovic, P., Finucane, M. L., Peters, E., \& MacGregor, D. G. (2004). Risk as analysis and risk as feelings: Some thoughts about affect, reason, risk, and rationality. Risk analysis, 24(311-322).

Sparbel, K. J. H., \& Anderson, M. A. (2000). Integrated literature review of continuity of care: Part 1, conceptual issues. Journal of nursing scholarship, 32(1), 17-24.

Toobert, D. J., Strycker, L. A., Glasgow1, R. E., Barrera, M., \& Bagdade, J. D. (2002). Enhancing support for health behavior change among women at risk for heart disease: The Mediterranean lifestyle trial Health Education Research, 17(5), 574-585.

Towle, B., C., \& Arslanoglu, T. (2003). Turkish-Americans. In D. Carroll (Ed.), Transcultural health care: A culturally competent approach (2nd ed.). Philadelphia: F.A. Davis Company.

Weinstein, N. D. (1999). What does it mean to understanding a risk? Evaluating risk comprehension. Journal of the National Cancer Institute. Monographs(25), 15-20.

Whittemore, R. (2005). The integrative review:Updated methodology. Journal of Advanced Nursing, 52(5), 546-553.

Wilcox, S., \& Stefanick, M. L. (1999). Knowledge and perceived risk of major diseases in middle-aged and older women. Health Psychology, 18(4), 346-353.

Wilson, D. K., Kliewer, W., Bayer, L., Jones, D., Welleford, A., Heiney, M., et al. (1999). The influence of gender and emotional versus instrumental support on cardiovascular reactivity in African-American adolescents Annals of behavioral medicine : a publication of the Society of Behavioral Medicine, 21(3), 235-243.

Witte, K. (1992). Putting fear back into fear appeals: The extended parallel processing model. Communication Monographs, 59(4), 329-349.

Witte, K., Meyer, G., \& Martell, D. (2001). Effective health risk messages: A step-by-step guide (1st ed.). Newbury Park, CA: SAGE.

Zabsonre, P., Sanou, G., Avanzini, F., \& Tognoni, G. (2002). Knowledge and perception of cardiovascular risk factors in Africa South of the Sahara. Archives des Maladies $d u$ Coeur et des Vaisseaux, 23-28. 


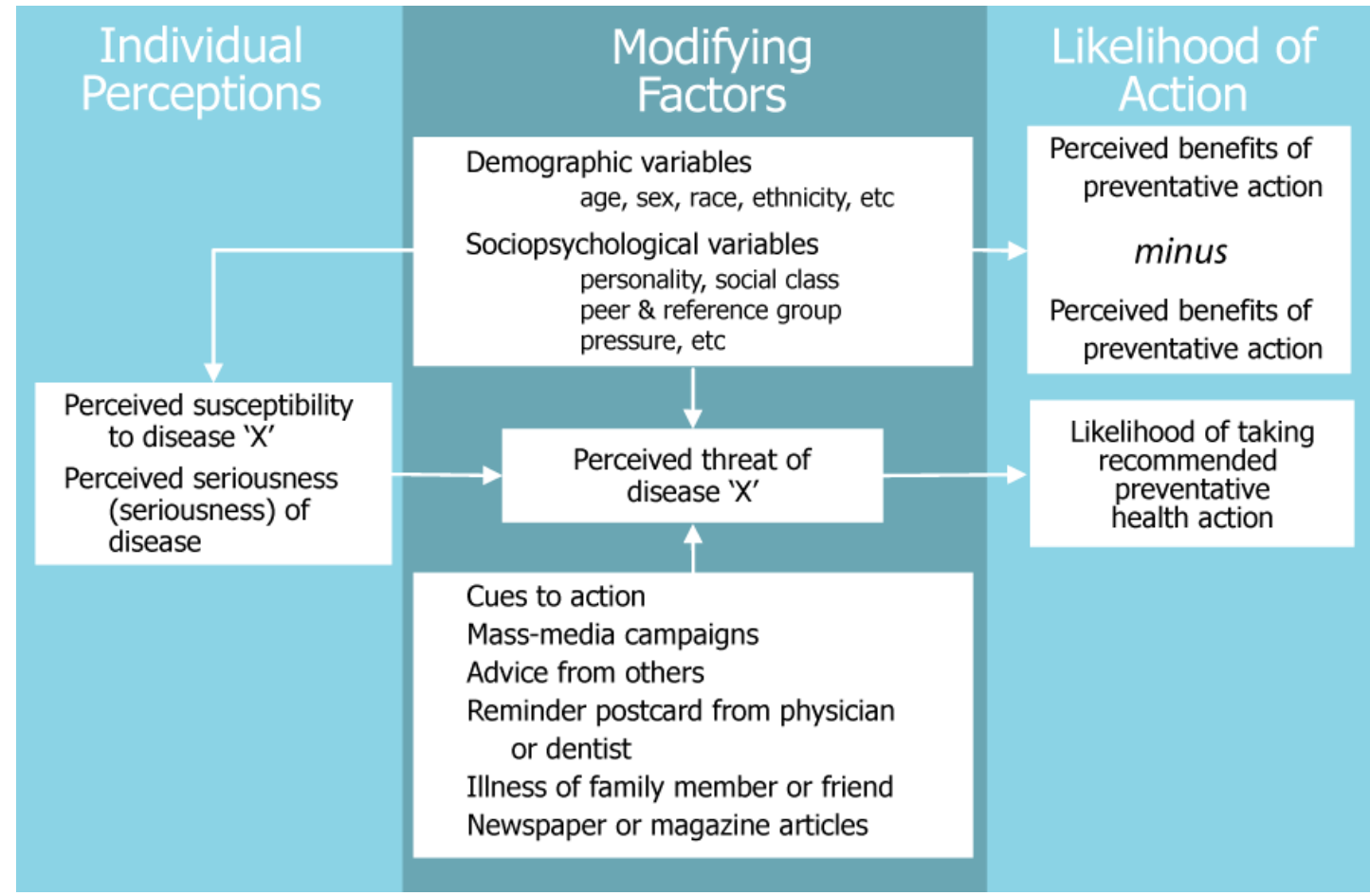

Figure 2.2. Health Belief Model

Source: (Glanz et al. 2002) 


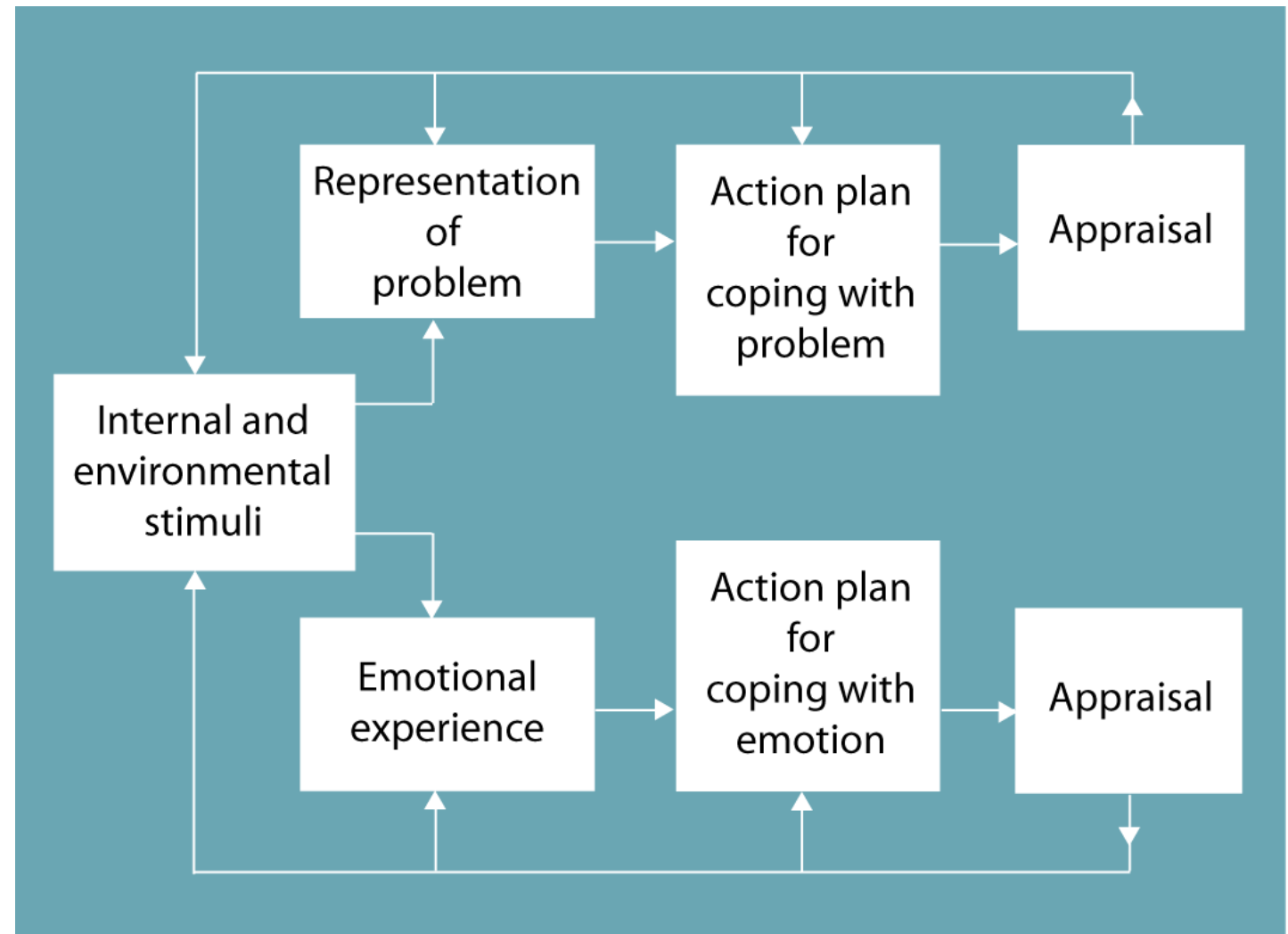

Figure 2.3. Leventhal's Self Regulatory Model

Source: (Leventhal et al. 1992) 


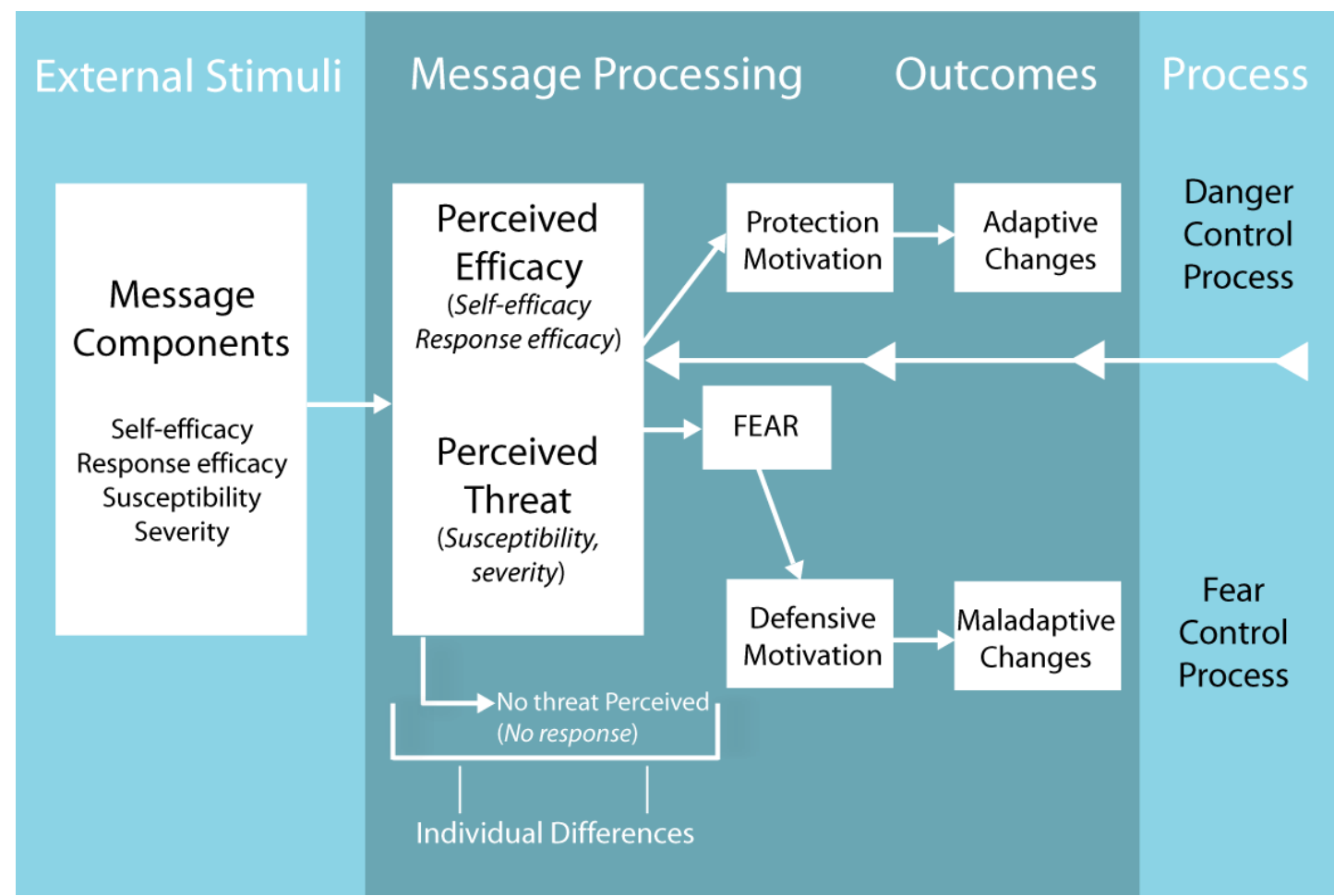

Figure 2.4. The Extended Parallel Process Model

Source: (Witte, 1992) 